\title{
Hypertensive renal disease
}

\author{
Doença renal hipertensiva
}

Ana Terezinha Guillaumon

Recent studies have shown a growing increase in renal stenosis; it is estimated that $6 \%$ of the adult population has hypertension. To date there have been few controlled epidemiological studies, but diagnosis rates of renal stenosis have increased, possibly because of improvements in the capacity to detect it, by both imaging exams and laboratory tests.

There are a range of different etiologies of hypertensive disease, including heart disease, renal artery stenosis and parenchymatous renal disease. Many authors have proposed simplified treatments, but knowledge of the underlying pathophysiology of disease and its repercussions for different organs is of fundamental importance to choosing a treatment and to whether that treatment will be successful.

Stenosis of the renal artery is caused by two important factors: degenerative diseases, such as progressive atherosclerosis, and inflammatory diseases, such as fibromuscular dysplasia. However, different age groups are predominantly affected by each type: the first, degenerative diseases, affects people over the age of 50, while the second prefers young people, particularly females. It is also important to consider the appearance of images in diagnosis, because atherosclerosis affects the origin of the renal artery, with atheromatous plaques that often begin in the aorta, whereas dysplasia affects the renal artery along its length, with an intense inflammatory process in the tunica media, producing an appearance of alternating stenosis and dilatation, somewhat like a bead necklace.

The first attempts to treat hypertension caused by stenosis of the renal artery took place during the 1970s, but patients who were treated using open surgery for revascularization of the organ suffered high rates of morbidity and mortality. It is believed that this was due to their advanced stage of kidney damage.

An alternative option, transluminal percutaneous angioplasty was first proposed around the same time and gradually refined, in terms of clinical and laboratory diagnosis, of imaging technologies and materials technologies, all of which made treatment easier and preservation of renal function more likely.
During the 1980s, it was found that endovascular surgery had surpassed the results of open surgery and nowadays the objectives are to control hypertension and preserve the kidney, reducing renal overload and achieving relative improvement of renal function. In some case, improvement is sufficient to avert a future need for enrolment on a hemodialysis program, which shortens survival. After the first results of treatment using angioplasty, it was found that placement of a stent reduced restenosis for long periods.

A ten-year follow-up study of patients with hypertensive renal disease caused by stenosis of the renal artery who were treated at a University Hospital following a predefined protocol observed that kidneys and renal function were preserved in $76 \%$ of cases. Although there are recent multicenter studies (CORAL, STAR, etc.) that contest these results that have been achieved and demonstrated, we believe that unsuccessful treatment outcomes are the result of erroneous etiologic diagnosis and disease staging. In view of this, it is necessary to study and analyze progression of kidney disease in order to intervene at the most appropriate point. When stenosis is significant, and there is at least a minimum of $30 \%$ of the renal parenchyma functioning, angioplasty/ stenting has a positive effect in reducing arterial blood pressure and removing renal overload, improving renal function. Therefore, the better preserved the parenchyma, the better the postoperative outcomes.

There are recent reports of treatment for renovascular hypertension using intravascular devices to produce denervation of the renal artery. The procedure is new and investigation of disease pathophysiology is of fundamental importance to application of this new technology. The results of some current randomized studies indicate that therapeutic effects are promising. However, more accurate studies are needed to define its indications, primarily in terms of their definitions of inclusion and exclusion criteria, but also analyzing larger samples, in order to specify for which types of hypertension (by etiology) we should be indicating this treatment. Considering that denervation of the renal artery leads to vasomotor paralysis due to removal of the effect of 
the sympathetic nervous system, in diseases where etiology is stenosis by vasoconstriction, this process can help to control hypertension, reduce left ventricle hypertrophy, increase glucose metabolism and be a possible cause of cardiac and renal failure in endstage disease; i.e. in a dependent vasoconstrictor process. A review of the results achieved in the 1960s and 1970s, when the same process was used in vessels of the lower limbs, found that aneurysms could form at denervation sites, possibly because the local sympathectomy destroyed the external layers of the vessel wall, reducing its strength. In view of the results of current studies, we therefore believe that renal artery ablation must be better investigated in wide-ranging and long-lasting studies, since it could prove to be an alternative antihypertensive treatment choice for patients resistant to current treatments. Furthermore, assessment and clinical management of patients must be studied exhaustively so that the method can be applied on the basis of the pathophysiologic and etiologic nature of the hypertension being treated.
Correspondence Ana Terezinha Guillaumon UNICAMP

Cidade Universitária “Prof. Zeferino Vaz", Barão Geraldo CEP 13083-887 - Campinas (SP), Brasi Fone: +55 (19) 3521-9450/Fax: +55 (19) 3521-8043

Author information Profa. Associada e Profa. Adjunto, Livre Docente da Faculdade de Ciências Médicas, Universidade Estadual de Campinas - UNICAMP, Departamento de Cirurgia, Faculdade de Ciências Médicas,

Campinas, SP, Brazil. 